circumvents cisplatin-resistance and is less dependent on p53 activity than cisplatin, these results suggest a molecular mechanism for VAL-083 that differs from both TMZ, BCNU and cisplatin. They further suggest that irreparable DNA damage induced by VAL-083 is impervious to common strategies employed by cancer cells to escape effects of alkylating drugs used in GBM treatment.

PS2 - 167

doi:10.1017/cjn.2016.365

CIC Deficiency is Associated with Dysregulation of Genes Involved in Cell Adhesion and Developmental Processes

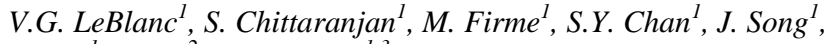
A. Lee, S. Yip ${ }^{2}$, M.A. Marra ${ }^{1,3}$

${ }^{I}$ BC Cancer Agency Genome Sciences Centre

${ }^{2}$ Departments of Pathology \& Laboratory Medicine

${ }^{3}$ Medical Genetics, University of British Columbia, Vancouver, BC vleblanc@bcgsc.ca

Somatic mutations in the Capicua (CIC) gene were first identified in Type I low-grade gliomas (LGGs), which are characterized by $1 \mathrm{p} / 19 \mathrm{q}$ co-deletions and IDH mutations. They are found at frequencies of $\sim 50-70 \%$ in this glioma subtype, and have since been identified in $\sim 40 \%$ of stomach adenocarcinomas (STADs) of the microsatellite instability (MSI) subtype; however, the role of these somatic mutations in malignancy has yet to be established. In Drosophila, CIC functions as a transcriptional repressor whose activity is inhibited upon activation of the mitogen-activated protein kinase (MAPK) signalling pathway. Though mammalian CIC appears to retain these functions, only three of its target genes have been established in human cells: ETV1, ETV4, and ETV5 (ETV1/4/5). To further probe CIC's transcriptional network, we developed CIC knockout cell lines and performed transcriptomic and proteiomic analyses in these and in control cell lines expressing wild type $C I C$, identifying a total of 582 differentially expressed genes. We also used RNA-seq data from The Cancer Genome Atlas (TCGA) for Type I LGGs and STADs to perform additional differential expression analyses between CIC-deficient and CIC-expressing samples. Though gene-level overlap was limited between the three contexts, we found that CIC appears to regulate the expression of genes involved in cell-cell adhesion, metabolism, and developmental processes in all three contexts. These results shed light on the pathological role of CIC mutations and may help explain why these have been associated with poorer outcome within Type I LGGs.

PS2 - 171

doi:10.1017/cjn.2016.366

\section{Bmi1 Identifies Treatment-Refractory Stem Cells in Human Glioblastoma}

P. Vora ${ }^{1}$, M. Qazi, C. Venugopal, M. Subapanditha,

S. Mahendram, C. Chokshi, M. Singh, D. Bakhshinyan,

N. McFarlane, S. Singh

${ }^{I}$ McMaster University, Hamilton, ON

pavora@mcmaster.ca

Glioblastoma (GBM) is an aggressive brain tumor that is resistant to conventional radiation and cytotoxic chemotherapies. We hypothesize that brain tumor initiating cells (BTICs), a subpopulation of treatment-resistant cells with stem cell properties cause tumor relapse and a subset of neural stem cell genes regulate BTIC self-renewal, driving GBM recurrence. We adapted the existing treatment protocol for adults with primary GBM for in vivo treatment of immunocompromised mice engrafted with GBMs. Post-chemoradiotherapy, the recovered GFP+ GBMs were profiled for self-renewal and expression of critical stem cell genes. Using invitro and invivo gain-of-function/loss-of-function experiments, we investigated the regulatory functions of Bmi1 in primary neural stem \& progenitor cells (NSPCs) and GBM tumor formation. Finally, global RNA-Seq profiling was performed to understand the consequences of Bmil dysregulation on target gene expression. GBM cells showed an increase in Bmil levels postchemoradiotherapy, suggesting the presence of a treatmentrefractory BTICs. GFP+ cells extracted from treated xenografts had higher self-renewal and BTIC marker expression. Although treated mice responded to therapy, we observed tumor relapse with increased Bmil expression. Knockdown of Bmil diminished selfrenewal and proliferation of GBM cells and delayed tumorigenesis, highlighting a critical role for Bmil in tumor maintenance. Conversely, over-expressing Bmi1 in NSPCs failed to initiate tumor formation in vivo. Using high-throughput sequencing data, we generated a map of signaling pathways dysregulated in GBM that may lead to tumor recurrence. Our data confirms the existence of a rare treatment-refractory BTIC population with enhanced selfrenewal capacity that escapes therapy and drives tumor relapse.

PS2 - 172

doi:10.1017/cjn.2016.367

\section{Preclinical Validation of a Novel CD33/CD3 Bispecific T-Cell Engager (BiTE) Antibody to Target Patient-Derived Glioblastoma Cells}

P. Vora ${ }^{l}$, C. Venugopal, C. Choksi, M. Qazi, J. Adams, M. London, M. Subapanditha, M. Singh, D. Bakshinyan, S. Sidhu, J. Moffat,

S. Singh

${ }^{I}$ McMaster University, Hamilton, ON

pavora@mcmaster.ca

Glioblastoma (GBM), an aggressive primary adult brain tumor, is feared for its near uniformly fatal prognosis. Despite the use of aggressive treatment including surgical resection, radiotherapy and chemotherapy, the outcome of patients with GBM has failed to improve significantly. Numerous studies have implicated CD133+ GBM subpopulation as driver of chemo- and radio-resistance. CD133 expression correlates with disease progression, recurrence, and poor overall survival of GBM patients. Here, we describe the preclinical evaluation of a recombinant CD133xCD3 bispecific Tcell engager (BiTE) antibody that redirects human polyclonal $\mathrm{T}$ cells to CD133+ GBM cells, inducing very potent anti-tumor response. CD133-specific BiTE was constructed; with one arm recognizing the tumor antigen (CD133) while the second is specific to CD3 antigen. Using CD133high and CD133low primary GBM lines, we validated the binding of BiTEs to CD133+ GBMs and $\mathrm{CD} 3+\mathrm{T}$ cells. In order to test the ability of BiTEs to functionally elicit CD133-specific cytotoxic responses in vitro, we performed killing assays. We observed CD133-specific BiTE mediated $\mathrm{T}$ cell activation and redirection to kill $\mathrm{CD} 133$ expressing GBM cells in a co-culture of T cells and GBM cells. The killing was more efficient in CD133high GBMs compared to CD133low GBMs, validating its specificity to target CD133+ BTICs. Treatment with BiTEs yielded significant reductions in 